\title{
Late post-operative endophthalmitis in a non-diabetic patient with urinary tract infection due to Candida albicans
}

\author{
M. Jayahar Bharathi, R. Ramakrishnan, C. Shivakumar \\ Department of Microbiology \& Molecular Biology, Aravind Eye Hospital \& PG Institute of \\ Ophthalmology, Tirunelveli, Tamil Nadu, India
}

\begin{abstract}
A 56-year-old women presented with redness, pain and diminution of vision in the right eye for the past month after having undergone cataract surgery six months earlier by phacoemulsification with implantation of a posterior chamber intraocular lens. No history of trauma, systemic or any ocular surface diseases were recorded. She had symptoms of photophobia and floaters. B-scan ultrasonography in the right eye demonstrated membrane echoes that were suggestive of endophthalmitis supported with thickened retinal choroid sclera complex. The intra-vitreal aspirate and urine specimen revealed presence of yeast-like fungus under direct microscopy and culture, while the blood, sputum and vaginal swab samples proved negative. Microbiological evaluation of ocular and other clinical specimens clearly confirmed that the source of infection could be attributed to the urinary tract being infected with $C$. albicans which could have arisen from contamination. This report fortifies the possible risk of urinary tract infection as a causative agent in post-operative endophthalmitis.
\end{abstract}

Key words: Post-operative endophthalmitis, Candida albicans, urinary tract infection, risk of post-cataract endophthalmitis

\section{Introduction}

Post-operative infectious endophthalmitis is one of the most devastating complications that can be encountered after any intraocular procedure, especially after a cataract surgery. ${ }^{1,2}$ The incidence, risk factors, prophylaxis, and management of endophthalmitis have been widely reported in ophthalmic literature. ${ }^{1-3}$ The ocular surface and adnexa acts as primary source for infectious agents in culture-positive cases of post-operative endophthalmitis. ${ }^{4}$ Many studies have demonstrated the predominance of bacteria as a cause of post-cataract surgical endophthalmitis, while fungal pathogens causing postoperative endophthalmitis are rare..$^{1-3}$ The yeast-like fungus Candida albicans has been reported as one of the common causes in endogenous endophthalmitis; ${ }^{3}$ endophthalmitis due to $C$. albicans of exogenous origin six months after the cataract surgery without any hematogenous dissemination in an immunologically healthy patient is rare. This case is analyzed in this report.

Correspondence: Dr. M. Jayahar Bharathi, Department of Microbiology \& Molecular Biology, Aravind Eye Hospital \& PG Institute of Ophthalmology, Tirunelveli, Tamil Nadu, India 627001. E-mail: jayahar@tvl.aravind.org 


\section{Case report}

A 56-year-old women presented with redness, pain, watering and diminution of vision in her right eye for the past month. Six months earlier, she had undergone an uncomplicated cataract surgery by phacoemulsification with implantation of a posterior chamber intraocular lens. There was no history of trauma, diabetes, hypertension, cardiac diseases or any ocular surface diseases. She had symptoms of photophobia and floaters. On examination, the best corrected visual acuity (VA) was 20/600 in the operated eye (right) and $20 / 30$ in the left eye. She had been using ofloxacin eye drops six times a day at the time of presentation. Slit-lamp biomicroscopic examination revealed grayish white exudates $(10 \mathrm{~mm} \times 6 \mathrm{~mm})$ in the inner lip of the corneal section and in the papillary area $(4 \mathrm{~mm} \times 3 \mathrm{~mm}$ ) of the anterior chamber adherent to the corneal endothelium of the right eye. Incidence of associated corneal edema, severe anterior chamber reaction and hypopyon $(0.5 \mathrm{~mm})$ were recorded. Aqueous flares and cells were both $3+$. Severe circumcorneal congestion was noted. Intraocular pressure was 20 and $18 \mathrm{mmHg}$ in the right and left eye respectively. Fundus details were obscured partially due to corneal edema and anterior chamber reaction, but appeared with the absence of red reflex. The chest X-ray was normal with the blood sugar level touching $75.0 \mathrm{mg} / \mathrm{dl}$ during fasting and $98.0 \mathrm{mg} / \mathrm{dl}$ postprandially.

B-scan ultra-sonography demonstrated membrane echoes suggestive of endophthalmitis that were supported with thickened retinal choroid sclera complex. A clinical diagnosis of the post-operative endophthalmitis was made and aqueous humor (AH) was aspirated, and intraocular antibiotic injections of vancomycin $(1 \mathrm{mg} / 0.1 \mathrm{ml})$ and amikacin $(400 \mu \mathrm{gm} / 0.1 \mathrm{ml})$ were given. The microbiological analysis of the $\mathrm{AH}$ showed negative for presence of any microbes. The patient was treated with $0.3 \%$ ciprofloxacin eye drops hourly and $1 \%$ cyclopentolate eye drops three times a day. Oral ciprofloxacin 750 $\mathrm{mg}$ twice a day, and oral prednisolone $70 \mathrm{mg} /$ day $(1 \mathrm{mg} / \mathrm{kg}$ ) were also administered. The left eye was normal. Based on the typical clinical findings of infectious endophthalmitis, IOL removal (due to the insta-

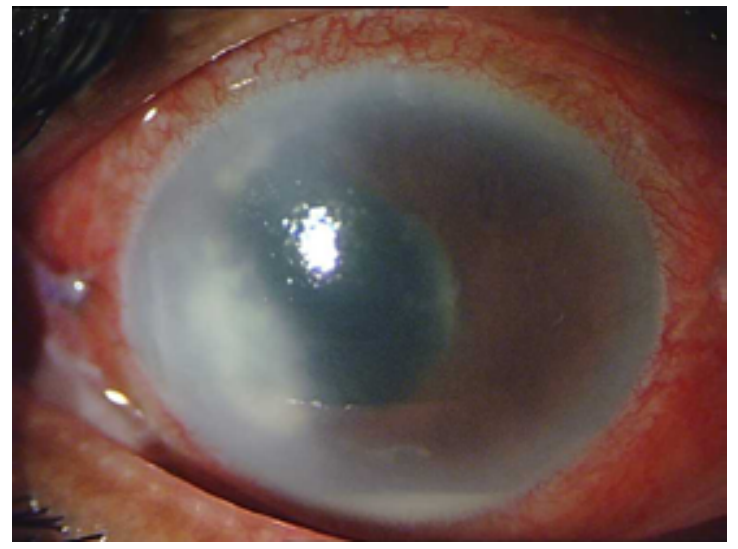

Fig. 1. Slit-lamp biomicroscopic photograph showing an inflamed eye with grayish white exudates $(10 \mathrm{~mm} \times 6$ $\mathrm{mm}$ ) extending from the inner lip of the corneal section extending up to the papillary zone $(4 \mathrm{~mm} \times 3 \mathrm{~mm})$ associated with hypopyon $(0.5 \mathrm{~mm})$ and vitreous exudates.

bility of the $\mathrm{IOL}$ ) and core vitrectomy were performed along with repeated aspiration of the $\mathrm{AH}$. The confirmatory diagnosis of infectious endophthalmitis was established by the microbiological evaluation of $\mathrm{AH}$ and vitreous fluid (VF), which were collected by using standard techniques. The part of the collected intraocular aspirates were immediately inoculated into the blood agar, chocolate agar, Sabouraud's dextrose agar and 


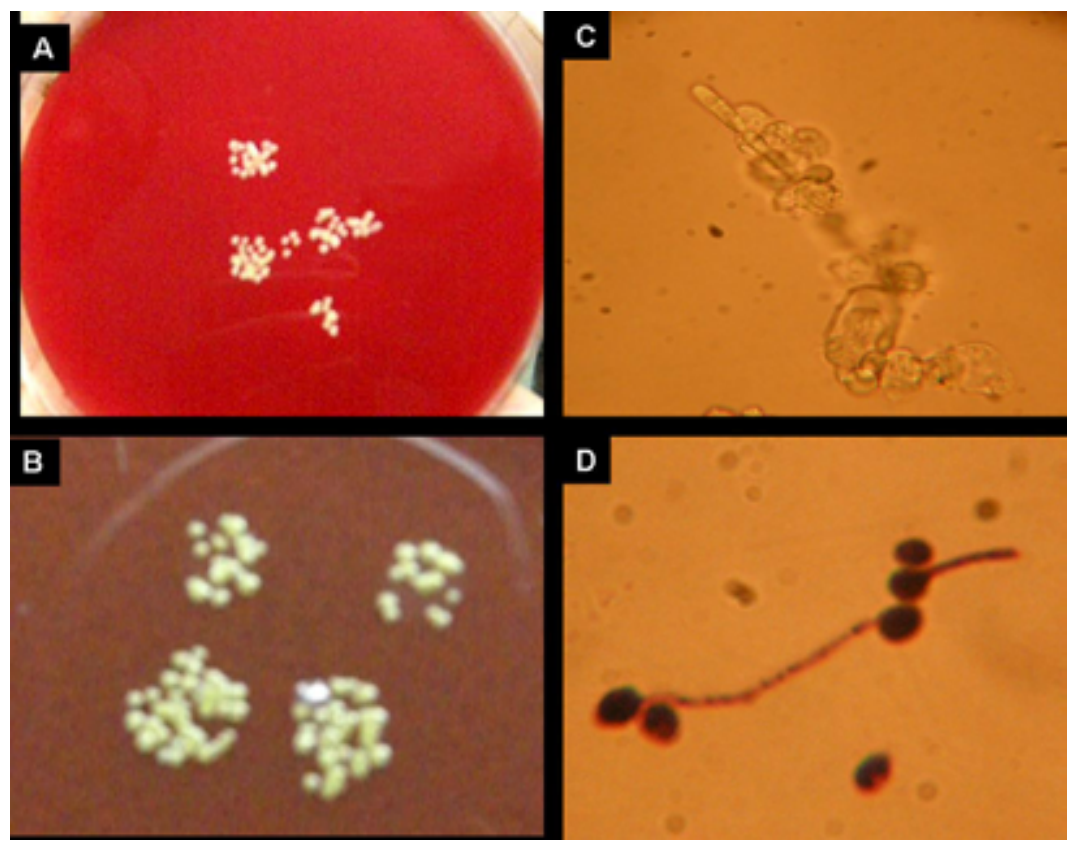

Fig. 2.

A. Candida albicans colonies at the inoculated sites on the blood agar plate inoculated with intra-vitreal aspirate.

B. Candida albicans colonies at the inoculated sites on the chocolate agar plate inoculated with intravitreal aspirate.

C. Vitreous aspirates mounted with $10 \%$ potassium hydroxide solution showing long pseudohyphae along with clusters of spherical shaped blastoconidia at the septa of a yeast-like organism.

D. Vitreous aspirate stained with the Gram-staining technique, showing long pseudohyphae along with clusters of spherical shaped blastoconidia at the septa of a yeast-like organism.

also into the liquid media- thioglycolate medium and brain-heart infusion broth in the operating room. ${ }^{5}$ The remaining part of the intraocular aspirates were smeared for $10 \%$ $\mathrm{KOH}$ wet mounting and Gram-staining procedures. ${ }^{5}$ In the early morning mid-stream urine, vaginal swab, sputum and blood specimens were collected and subjected to microbiological analysis using standard protocols., ${ }^{6,7}$ The inoculated plates for bacterial isolation were incubated both aerobically and anaerobically, while the fungal Sabouraud's dextrose agar plates were incubated under biochemical oxygen demand. The $10 \% \mathrm{KOH}$ wet mounting and gram-stained smear of $\mathrm{AH}, \mathrm{VF}$ and urine specimen were found to be positive for a yeast-like fungus. Similarly, the culture of $A H, V F$ and urine specimen were also found to be positive for (significant) Candida albicans growth. The blood specimen showed no growth, while the sputum specimen was negative for the growth of Candida species even after seven days after the incubation. Standardized microbiological evaluation protocols were followed for ocular and non-ocular clinical specimens..$^{5-7}$ The pathological analysis of peripheral blood smear showed a normal blood picture and no evidence for immuno-competency disorders or parasitic 
infections. After the yeast-like fungus (Candida sp.) was seen in smears of AH and VF without the presence of bacteria, the treatment regimen was changed to intra-vitreal voriconazole twice and topical $0.15 \%$ amphotericin B and $1 \%$ voriconazole topically on an hourly basis. In spite of intensive antifungal therapy, the vision in that eye could not be restored, resulting in phthisis.

\section{Discussion}

The microbial etiology of endophthalmitis varies with geographical location and also from person to person depending on the microbial flora at the ocular surface..$^{1-3}$ The most common infecting organism of the post-operative endophthalmitis following cataract extraction are the coagulase-negative Staphylococcus species. ${ }^{1,2,3} \mathrm{Gram}$ negative bacteria and anaerobes are much less frequent causative agents. ${ }^{1-3} \mathrm{~A}$ review of the literature showed that fungal endophthalmitis is common among patients with systemic debilitating diseases, malignancy, intravenous drug use, chemotherapy, systemic antibiotics and prolonged corticosteroids therapy, alcoholism and diabetes. ${ }^{8}$ However, filamentous fungi are also frequently encountered among traumatic endophthalmitis and endophthalmitis due to fungal keratitis, while the yeast-like fungi Candida species are common among endogenous endophthalmitis in cases with candidemia. ${ }^{9}$ Candida endophthalmitis and disseminated candidiasis usually occurs in patients with parental drug abuse and in severely ill patients whose immune mechanisms have got altered.

Candida species are part of the human flora; they exist commensally. When the immune system is compromised, these organisms become potentially pathogenic. ${ }^{8-10}$ In the present case, endophthalmitis occurred six months after the post-operative period due to C. albicans which was recovered from two of the four cultured specimens, viz., vitreous aspiration and urine. Blood culture was negative and hence the evidence for candidemia or hematogenous dissemination of Candida to the eye was eliminated. Higher rates of positive urine cultures have been reported in candidemia and immunocompromised cases. ${ }^{8}$ It is construed from the study that the risk of developing endophthamitis could arise from urinary tract infections harboring C. albicans which could be ascribed to contamination through improperly cleaned after urination, or unclean hands, that might have acted as potent carriers. The continuous contact of the operated eye and its adnexal structures with the contaminated hands carrying C. albicans could be the primary cause of eye infection in our case. This report documents and fortifies the risk of urinary tract infection as a causative agent in post-operative endophthalmitis.

\section{References}

1. Lemley CA, Han DP. Endophthalmitis: A review of current evaluation and management. Retina 2007;27:662-680.

2. Ramakrishnan R, Bharathi MJ, Shivkumar C, Mittal S, Meenakshi R, Khadeer MA, et al. Microbiological profile of culture-proven cases of exogenous and endogenous endophthalmitis: a 10-year retrospective study. Eye 2009;23:943-956.

3. Kresloff MS, Castellarin AA, Zarbin MA. Endophthalmitis. Surv Ophthalmol 1998;43:193-224.

4. Speaker MG, Milch FA, Shaf MK, Eisner W, Kreiswirth BN. Role of external bacterial flora in the pathogensis of acute postoperative endophthalmitis. Ophthalmology 1991;98:639-649. 
5. Wilhelmus KR, Liesegang TJ, Osato MS, Jones DB. Cumulative Techniques and Procedures in Clinical Microbiology 13A, Laboratory Diagnosis of Ocular Infections. Washington DC: American Society for Microbiology, 1994.

6. Clarridge J, Johnson JR, Pezzlo M. Cumulative Techniques and Procedures in Clinical Microbiology 2B, Laboratory Diagnosis of Urinary Tract Infections. Washington DC: American Society for Microbiology, 1998.

7. Dunne WM, Nolte FS, Wilson M. Cumulative Techniques and Procedures in Clinical Microbiology $1 B$. Blood Cultures III. Washington DC: American Society for Microbiology, 1997.

8. Chakrabarti A, Shivaprakash MR, Singh R, Tarai B, George VK, Fomda BA, Gupta A. Fungal endophthalmitis: Furteen years' experience from a center in India. Retina 2008;28:1400-1407.

9. Donahue SP, Craig MG, Zuravleff JJ, Eller AW, Nguyen MH, Peacock JE Jr, et al. Intraocular candidiasis in patients with candidemia. Clinical implications derived from a prospective multicenter study. Ophthalmology 1994;101:1302-1309.

10. Rensch F, Schlichtenbrede FC, Jonas JB. Postoperative mycotic endophthalmitis. J Cataract Refract Surg 2010;36:1233-1234. 